\title{
Implementación del Estándar ISO/IEC 29110 en Centros de Desarrollo de Software de Universidades Mexicanas: Experiencia del Estado de Zacatecas
}

\author{
Mirna Muñoz ${ }^{1}$, Jezreel Mejia ${ }^{1}$, Claude Y Laporte ${ }^{2}$ \\ mirna.munoz@cimat.mx,jmejia@cimat.mx, claude.Laporte@etsmtl.ca \\ ${ }^{1}$ Centro de Investigación en Matemáticas, Avda. Universidad no. 222, 98068, Zacatecas, México \\ ${ }^{2}$ École de technologie supérieure, Department of Software and IT Engineering, 1100, Notre-Dame Street \\ West, Montréal, Québec, Canada
}

DOI: 10.17013/risti.29.43-54

\begin{abstract}
Resumen: Hoy en día las empresas muy pequeñas de desarrollo de software tienen una gran presencia en la industria del software en cualquier país, donde alrededor del 94\% está formado por este tipo de empresas. Esta situación resalta la creciente necesidad de mejorar sus procesos de desarrollo de software que les permitan desarrollar productos y servicios de calidad. Para lograrlo se deben cumplir dos requisitos: (1) integrar una cultura de procesos y mejora continua en las organizaciones, y (2) dotar de personal altamente cualificado que tenga el conocimiento y habilidades para trabajar exitosamente con modelos y/o estándares utilizados en las organizaciones. Este artículo presenta la experiencia de la implementación del estándar ISO/IEC 29110 para reforzar el proceso de desarrollo de software de cuatro centros de desarrollo de software de universidades mexicanas como una solución para abordar con los dos requisitos previamente mencionados.
\end{abstract}

Palabras-clave: estándar ISO/IEC 29110; ingeniería de software; centro de desarrollo de software; empresas muy pequeñas; cultura de procesos.

\section{Implementation of ISO/IEC 29110 in Software Development Centers from Mexican Universities: An experience of the Zacatecas State}

\begin{abstract}
Nowadays the very small organizations of software development have a great presence in the software development industry, because in most countries about $94 \%$ is made up of very small organizations. This situation highlights the increasing need for improving their development software processes that allows them to develop quality products and services. To achieve it, two requirements should be covered: (1) integrate a process culture and continuous improvement in organizations and (2) provide high quality personnel with knowledge and abilities for working successfully under models and standards used in organizations. This paper presents an experience in the implementation of the ISO/IEC 29110 standard to reinforce the software development process of four software development
\end{abstract}


centers from mexican universities as a solution to cover the requirements before mentioned.

Keywords: ISO/IEC 29110 standard; software engineering; software development center; very small entities; process culture.

\section{Introducción}

Hoy en día la mayor parte del software a nivel mundial es desarrollado por empresas muy pequeñas, generando una gran oportunidad para este tipo de empresas (Muñoz, Negrón, Mejia, \& Lopez, 2018), por lo tanto, dos principales desafíos en esta área que se hacen presentes son, por un lado, reforzarlas con el desarrollo de modelos y/o estándares ad hoc que cubran la creciente necesidad de apoyarlas en la implementación de buenas prácticas de ingeniería de software para el desarrollo de productos de calidad que les permitan mejorar su operación y sus procesos (Sánchez-Gordon, de Amescua, O'Connor, \& Larrueca, 2017) (Larrucea, O’Connor, Colomo-Palacios, \& Laporte, 2016). Por otro lado, que las universidades sean capaces de responder a las demandas de formación de personal altamente cualificado para el desarrollo de software de alta calidad (Muñoz, Peña, Mejia, \& Lara, 2016).

Como una respuesta a esta necesidad surge el estándar ISO/IEC 29110, que lleva como título "Ingeniería de software - Perfiles de ciclo de vida de entidades muy pequeñas", el cual comprende una serie de directrices e informes técnicos enfocados en apoyar a las empresas muy pequeñas a incrementar la calidad de sus productos y servicios, mientras incrementan su productividad y mejoran sus procesos con costos mínimos (Sánchez-Gordon et al., 2017) (Larrucea et al., 2016). Además de minimizar la brecha en el uso de buenas prácticas en empresas que carecen de conocimiento o experiencia práctica en la implementación de modelos de procesos y/o estándares (Laporte, Muñoz \& Gerançon, 2017).

Este artículo presenta la experiencia en la implementación del estándar ISO/IEC 29110 en Centros de Desarrollo de Software (CDS) de Universidades del Estado de Zacatecas, México para reforzar el proceso de desarrollo de software. La finalidad de la implementación del ISO/IEC 29110 en las universidades es lograr que los estudiantes tengan experiencia en el uso de buenas prácticas en la realización de proyectos reales. Esta acción cubre los dos retos principales mencionados en (Muñoz et al., 2016) a los que se enfrentan los recién graduados al integrarse a la industria del software: 1) tener el conocimiento para trabajar con roles en el desarrollo de un proyecto, por ejemplo, gestores de proyecto, gestor de calidad o ser parte del equipo de desarrollo y 2) tener la experiencia de trabajar bajo modelos y estándares de calidad.

Después de la introducción el artículo está estructurado como sigue: en la sección 2 se describen conceptos clave, así como los antecedentes del trabajo presentado; en la sección 3 se muestra el proceso seguido para reforzar al CDS mediante la implementación del estándar ISO/IEC 29110 en los CDS's de las universidades; en la sección 4 se presentan los resultados en los 4 CDS's al implementar el estándar; y en la sección 5 se muestran las discusiones y conclusiones. 


\section{Antecedentes}

\subsection{Conceptos clave}

1. Centro de desarrollo de software (CDS): es un centro de trabajo dedicado al desarrollo de software, cuyo objetivo es aumentar la productividad, disminuir costos y elevar la calidad de productos y servicios ofrecidos a clientes, donde el desarrollo de software es realizado como un proceso industrial, esto es definible, repetible y medible en un entorno de alta productividad. En México, empresas como Softeck, Certum e IBM centro de diseño y desarrollo de software han implementado este tipo de centros de trabajo con resultados exitosos (Software Gurú, 2018).

2. Estándar ISO/IEC 29110: proporciona una serie de guías y directrices desarrollados para mejorar el proceso de desarrollo de software de las entidades muy pequeñas, ayudándolas en la implementación de buenas prácticas para la obtención de beneficios como incremento en la calidad del producto y/o servicio, reducción en tiempos de entrega y reducción en costos de producción (Laporte, et al., 2017).

Entre las principales características del estándar se pueden mencionar: (1) está compuesto por 4 perfiles (perfil de entrada, perfil básico, perfil intermedio y perfil avanzado) que pueden ser usados por las entidades muy pequeñas de acuerdo a sus objetivos; (2) comprende dos categorías de procesos, el proceso de gestión de proyectos y el proceso de implementación de software; (3) puede ser utilizado independientemente del enfoque de desarrollo o metodología utilizado y (4) proporciona un conjunto de elementos de proceso que facilitan su adopción como son objetivos, tareas, roles y productos de trabajo (Laporte, et al., 2017). El perfil básico del estándar está compuesto por dos procesos: el proceso de Gestión de Proyectos (GP) y el proceso de Implementación de Software (IS) descritos a continuación. Además, en la Figura 1 se incluye una descripción global de su funcionamiento.

Los procesos abarcados en estándar ISO/IEC 29110 son dos (Laporte, et al., 2017), como a continuación se listan:

- Proceso de gestión de proyectos: establece y lleva a cabo tareas relacionadas con la gestión del proyecto de forma sistematizada, tal que, se cumpla con los objetivos del proyecto. Las actividades que contiene este proceso son cuatro: planificación del proyecto, ejecución del plan del proyecto, evaluación y control del proyecto, y cierre del proyecto.

- Proceso de implementación de software: ejecuta de manera sistematizada las actividades relacionadas con el desarrollo de software (análisis, diseño, construcción, integración y pruebas), todas de acuerdo a los requisitos especificados para el desarrollo de nuevos productos o la modificación de nuevos productos. Las actividades que contienen este proceso son seis: inicio, análisis, diseño, construcción, integración y pruebas, y entrega. 


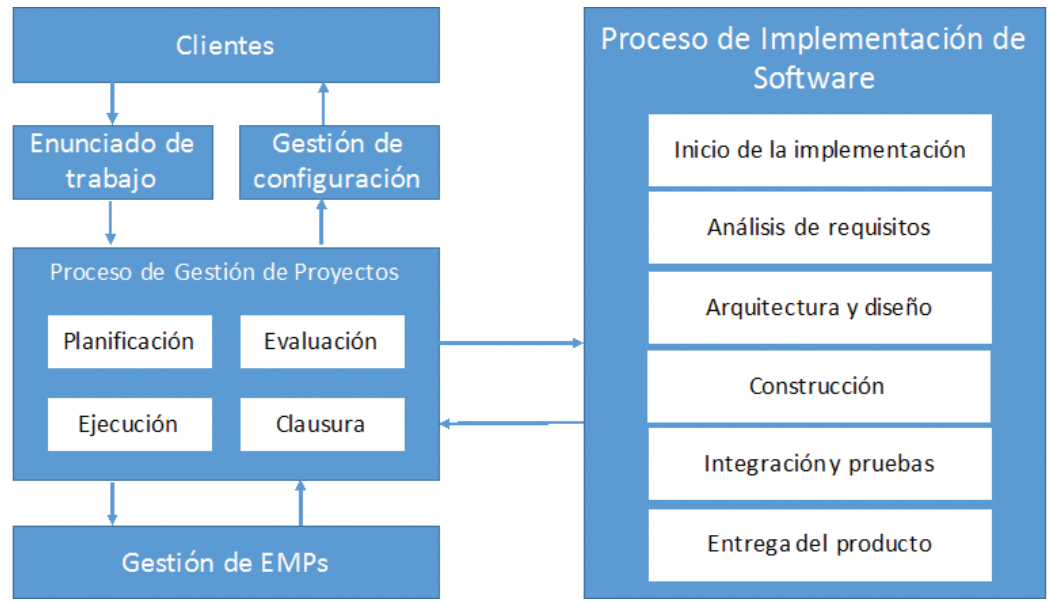

Figura 1 - Visión global de los procesos del ISO/IEC 29110 del perfil básico (Laporte et al., 2018)

Como se observa en la Figura 1, el enunciado de trabajo del cliente es el que se utiliza para iniciar el proceso de gestión de proyectos. El plan del proyecto es utilizado como guía para la ejecución de las actividades de análisis de requisitos, la arquitectura de software y diseño detallado, construcción del software, integración y pruebas de software y liberación producción. El proceso de gestión de proyectos, posteriormente, cierra las actividades de entrega de la configuración del software (que es, el conjunto de productos de software) y obtienen la aceptación del cliente para formalizar la finalización del proyecto.

Finamente, cabe resaltar que los procesos están definidos utilizando un conjunto de elementos del proceso que facilitan su implementación. La Tabla 1 presenta a manera de ejemplo el detalle en el que está definido cada actividad del proceso en la guía de gestión e ingeniería del perfil básico del software. Como se observa en la tabla, en la primera columna, se listan los roles que intervienen en la tarea que son el cliente (CUS) y el analista (AN); en la segunda columna, se describen las tareas; en la tercera columna, se lista los productos requeridos para ejecutar la tarea, y en la cuarta columna, se listas los dos productos de trabajo que se obtienen al ejecutar la actividad.

\begin{tabular}{llll}
\hline Roles & Lista de Tareas & $\begin{array}{l}\text { Productos de } \\
\text { trabajo de entrada }\end{array}$ & $\begin{array}{l}\text { Productos de } \\
\text { trabajo de salida }\end{array}$ \\
\hline & $\begin{array}{l}\text { SI2.4 Validar y obtener aprobación de la } \\
\text { especificación de requisitos. }\end{array}$ & $\begin{array}{l}\text { Especificación de } \\
\text { requisitos (validada) }\end{array}$ \\
Cus & $\begin{array}{l}\text { Validar que la especificación de requisitos las } \\
\text { necesidades y expectativas convenidas. } \\
\text { AN }\end{array}$ & $\begin{array}{l}\text { Los resultados encontrados se documentan } \\
\text { en los resultados de validación de resultados } \\
\text { y las correcciones son realizadas hasta que el } \\
\text { documento se apruebe por el cliente }\end{array}$ & $\begin{array}{l}\text { requisitos (verificada) } \\
\text { Resultados de la }\end{array}$ \\
\hline
\end{tabular}

Fuente: ISO/IEC Management and engineering guide: Generic profile group: Basic profile.

Tabla 1 - Ejemplo de una tarea de la actividad del análisis de requisitos (ISO, 2012) 
Es importante mencionar que los procesos de gestión de proyectos e implementación de software definidos en la guía de gestión e ingeniería de software (ISO, 2012), pueden ser usados como procesos “tal cual” están definidos en pequeñas organizaciones que no tengan procesos establecidos.

3. Entidades muy pequeñas: puede ser una empresa pequeña, un área, un proyecto o un equipo de trabajo en una empresa grande que desarrollan software y que tienen hasta 25 personas (Laporte \& O’Connor, 2016).

\subsection{Antecedentes en México}

México ha tenido una participación activa en modelos y estándares de calidad tanto en su desarrollo (modelo Moprosoft) como en su difusión (modelo CMMI-DEV y estándares ISO). Además, el gobierno mexicano ha establecido en su agenda nacional como una de las actividades reforzar a las empresas mexicanas para hacerlas competitivas a nivel internacional en este tipo de modelos y/o estándares (Laporte, et al., 2017).

Actualmente uno de los estándares adecuados para su implementación dentro de las muy pequeñas empresas de desarrollo de productos y/o servicios de software que representan en promedio el 90\% en México es el estándar ISO/IEC 29110. Con respecto a este estándar, en el 2006 fue presentado por los delegados mexicanos el modelo Moprosoft al grupo de trabajo 24 (WG24, por sus siglas en inglés) de la ISO. Posteriormente, este grupo decidió la adaptación de Moprosoft para cubrir los aspectos de las empresas muy pequeñas, tomando como base para la creación del ISO/IEC 29110 (Laporte, et al., 2017).

En este contexto el gobierno del estado de Zacatecas, México, ha desarrollado una estrategia para incrementar la competitividad de las empresas que esté alineada a la agenda nacional definiendo dos actividades para lograr tal fin:

1. Lograr que las empresas muy pequeñas incrementen el nivel de capacidad de sus procesos a nivel nacional: esta actividad hace referencia al incremento de la capacidad de los servicios/productos TI mediante la adopción de modelos y estándares que sirvan como referencia para la adopción de buenas prácticas de ingeniería de software que apoyen a las organizaciones a incrementar la productividad y calidad de las organizaciones.

2. Incrementar la calidad y cantidad de talento relacionado con el desarrollo de software y servicios de TI: esta actividad hace referencia a disponibilidad de capital humano mejor preparado, tal que, se pueda aprovechar el crecimiento del sector TI tanto a nivel local, así como para acceder a mercados internacionales.

Este artículo está relacionado con la segunda actividad, ya que se enfoca en los CDS's de 4 Universidades de la región de Zacatecas, en las que se están formando ingenieros relacionados con el desarrollo de software.

\section{Método Seguido para la Implementación del Estándar ISO/IEC 29110 en los Centro de Desarrollo de Software}

Para llevar a cabo la implementación del estándar se definió un método de seis pasos como se menciona a continuación: 
1. Formar a los CDS's en el ISO/IEC 29110 mediante la realización de workshops para la formación de los CDS's: por una parte, se desarrolló un workshop de concientización en el estándar, impartido por uno de los autores de este artículo. Además, los CDS's estuvieron en el curso de capacitación impartido por la empresa certificadora en el estándar.

2. Identificar y formalizar sus buenas prácticas: se realizó una reunión en la que el CDS mostró su forma de trabajo, tanto para el proceso de gestión de proyectos como para el proceso de implementación de software.

3. Identificar los principales problemas que tienen los CDS's en la ejecución de sus proyectos como normalmente lo realizan: se realizó una revisión de un proyecto del CDS en el que se identificaron "huecos" o incongruencias tanto en los procesos de gestión de proyectos como de implementación del software. Posteriormente, se preguntaron dudas sobre "huecos" en prácticas o documentos, teniendo retroalimentación de estos.

4. Mapear los procesos del CDS con los procesos del perfil básico del estándar ISO/ IEC 29110: una vez identificadas las buenas prácticas se realizó una trazabilidad teniendo en cuenta elementos de proceso como: actividades, lista de tareas, productos de entrada y productos de salida. Además, cada producto de entrada y salida se trazó con un checklist de elementos de cada producto.

5. Seleccionar y adaptar prácticas proporcionadas por el estándar al contexto del CDS: resultado del mapeo y con base en el cumplimiento de los objetivos de cada proceso, se seleccionaron aquellas prácticas clave proporcionadas por el estándar, que reforzarían al CDS en el cumplimiento de los procesos.

6. Revisar el proyecto del CDS en el cual se estén implementando las prácticas del estándar, reportar las no conformidades identificadas y proporcionar retroalimentación: se mantuvieron reuniones para revisar las actividades implementadas para el proceso de gestión de proyecto e implementación de software para detectar las no conformidades relacionadas con lo requerido por el estándar.

Cabe resaltar que la ejecución de los pasos del 2 al 6 se llevaron a cabo en promedio 6 reuniones por CDS.

\section{Resultados}

Esta sección presenta los resultados de los 4 centros de desarrollo de software describiendo los beneficios detectados. Cabe resaltar que los 4 CDS's fueron sometidos a un proceso de certificación formal por parte de la empresa certificadora del estándar ISO/IEC 29110 en México, lográndose certificar en el mismo.

\subsection{Centro de Desarrollo de Software de una Institución de Educación Superior del Sur del Estado de Zacatecas}

Es un centro de desarrollo de software constituido dentro de una educación superior para el desarrollo de proyectos de software para clientes externos e internos a la institución. 
Los equipos de desarrollo de proyectos están integrados por estudiantes y un profesor para el seguimiento del progreso del proyecto. Para el desarrollo de sus proyectos, los equipos siguen un proceso híbrido basado en CMMI y la metodología Scrum.

El proyecto en el cual se implementaron prácticas del ISO 29110 es un sistema de escritorio para la gestión de la operación y control de la información de un gimnasio, con la finalidad de hacer este proceso más eficiente.

Los beneficios detectados con la implementación de buenas prácticas sugeridas en el estándar se listan a continuación:

- La implementación de controles a través del proceso de desarrollo de software (CDS1-B1).

- Una mayor confianza de que se realiza la documentación requerida en un proceso de desarrollo (CDS1-B2).

- Una mayor confianza en la obtención de la aprobación de las partes interesadas sin asumir "cosas" en las que debe de haber un acuerdo (CDS1-B3).

- Una mejor gestión de los compromisos del equipo de desarrollo y el cliente (CDS1-B4).

\subsection{Centro de Desarrollo de Software de una Universidad de Zacatecas}

Es un centro de desarrollo de software constituido como parte de la universidad, cuyo objetivo es el desarrollo de proyectos de software para clientes externos e internos a la institución.

Los equipos que desarrollan los proyectos están integrados por un estudiante y un profesor, cuya tarea se enfoca en el seguimiento del progreso del proyecto. Cabe resaltar que, para el desarrollo de sus proyectos, los equipos siguen metodologías como TSP o Scrum dependiendo de las necesidades de control requeridas para el proyecto, así como las necesidades de control del cliente.

El proyecto en el cual se implementaron prácticas del estándar ISO 29110 es para la optimización de funcionalidad del sistema de calidad ambiental y sostenibilidad (DGCAMS) para la universidad.

Los beneficios detectados con la implementación de buenas prácticas sugeridas en el estándar se listan a continuación:

- La implementación de un estándar fácil de seguir para la gestión de un proyecto de desarrollo de software (CDS2-B1).

- La adopción de una forma estructurada para llevar a cabo un proceso de desarrollo de software que permite la mejora continua (CDS2-B2).

- El desarrollo de mínima documentación que facilitan tanto la gestión del proyecto como la documentación del software (CDS2-B3).

- Adopción de una mejor manera de controlar las versiones de un proyecto, con la implementación de un estándar de nomenclatura para la documentación de proyectos (CDS2-B4).

- Adopción de una forma estructurada de trabajar que facilita la sistematización de la ejecución del proceso (CDS2-B5). 


\subsection{Centro de Desarrollo de Software de una Institución de Educación Superior del Norte del Estado de Zacatecas}

Es un centro de desarrollo de software constituido dentro de un instituto de educación superior para el desarrollo de proyectos de software para clientes externos e internos a la institución.

Los equipos de desarrollo de proyectos están integrados por estudiantes y uno o dos profesores para el seguimiento del progreso del proyecto. Para el desarrollo de sus proyectos, los equipos siguen la metodología Scrum.

El proyecto en el cual se implementaron prácticas del ISO 29110 es un sistema de control de inventarios.

Los beneficios detectados con la implementación de buenas prácticas sugeridas en el estándar se listan a continuación:

- Mejora en las actividades relacionadas con el monitoreo y control del proyecto (CDS3-B1).

- Mejora en la comunicación con el cliente mediante la firma de acuerdos y la adopción de buenas prácticas de la definición de instrucciones de entrega del producto (CDS3-B2).

- Mejora en la gestión de riesgos (CDS3-B3).

- Mejora en la gestión de requisitos de software (CDS3-B4).

- Mejora en el análisis y diseño de software (CDS3-B5).

- Mejora en las actividades relacionadas con la validación y verificación de elementos del proyecto, por ejemplo, las pruebas de software (CDS3-B6).

\subsection{Spin-off de una institución de educación superior en la Ciudad de Zacatecas}

Es un Spin-off constituido dentro de un instituto de educación superior para el desarrollo de proyectos de software para clientes externos e internos a la institución.

Los equipos de desarrollo de proyectos están integrados por estudiantes y un profesor para el seguimiento del progreso del proyecto. Para el desarrollo de sus proyectos, los equipos siguen la metodología Scrum.

El proyecto en el cual se implementaron prácticas del ISO 29110 es un sistema para gestión y control de un laboratorio de lenguajes.

Los beneficios detectados con la implementación de buenas prácticas sugeridas en el ISO/IEC 29110 se listan a continuación:

- Genera un proceso de mejora continua para la optimización de la calidad de los productos de software (CDS4-B1).

- Establecimiento de un conjunto de plantillas que ayudan en la implementación de un proceso de desarrollo de software en un tiempo más corto, que permite a los estudiantes avanzar más rápido en su proceso de aprendizaje (CDS4-B2).

- Desarrollo de una herramienta para la evaluación del estado de las actividades, que permite tener un mejor control de las actividades (CDS4-B3) 


\section{Discusión y Conclusiones}

En (Muñoz et al., 2016b) se menciona un conjunto de deficiencias detectadas en 15 empresas pequeñas, cuando éstas contratan nuevo personal:

- Deficiencias de conocimiento: 14 mencionaron deficiencias en la gestión de proyectos; 12 mencionaron deficiencias en actividades relacionadas con el aseguramiento de calidad y 10 mencionaron deficiencias en el uso de modelos y estándares de calidad.

- Deficiencias en habilidades: la deficiencia mayormente mencionada esta relacionada con la toma de decisiones, seguida de falta de habilidades de gestión de riesgos y capacidades para resolver problemas y de trabajo en equipo.

De acuerdo con los beneficios identificados en el análisis de los 4 CDS's, estas deficiencias pueden ser fácilmente reducidas con la implementación del ISO/IEC 29110. En la Tabla 2 se muestra un análisis en el que se mapean deficiencias detectadas por las empresas, tanto deficiencias de conocimiento (DC), cómo deficiencias de habilidades (DH) y los beneficios obtenidos por los CDS's mediante la implementación del estándar (ver sección 4, subsecciones de 4.1 a 4.4).

Como se observa en la Tabla 2 al realizar la trazabilidad entre los tres elementos, las deficiencias detectadas en las empresas son cubiertas al implementar buenas prácticas de ingeniería de software enfocadas en la gestión de proyectos (proceso de gestión de proyectos) y el desarrollo de software (proceso de implementación de software), permitiendo además que los estudiantes desarrollen habilidades requeridas por la industria mexicana como son la toma de decisiones, gestión de riesgos, capacidad para resolver problemas y el trabajar en equipo.

Con base en los resultados antes mencionados, se puede concluir que el perfil básico del estándar ISO / IEC 29110 proporciona un estándar aceptado en la industria mexicana del software, dato que se confirma al revisar el padrón de empresas certificadas en el estándar ISO/IEC 29110 publicado por NYCE en (NYCE, 2011), donde se menciona que 31 de las 38 empresas certificadas en el estándar son mexicanas.

Este artículo presentó la experiencia de reforzar 4 CDS's utilizando tanto la metodología Scrum, como un proceso híbrido entre CMMI y Scrum, con la implementación del estándar ISO / IEC 29110.

Los resultados demuestran que con la implementación del estándar ISO/IEC 29110 es posible cubrir con los dos requisitos planteados en el resumen de este artículo. En primer lugar, la integración de una cultura de procesos y mejora continua en las organizaciones, ya que es fácilmente adaptable independientemente de la metodología o ciclo de desarrollo utilizado por la organización, además de promover la mejora continua.

En segundo lugar, es posible formar en las universidades personal altamente cualificado que tenga el conocimiento y habilidades para trabajar exitosamente con modelos y/o estándares utilizados en las organizaciones, ya que el CDS ofrece la oportunidad a los alumnos de la adopción, implementación y uso de buenas prácticas para el desarrollo de proyectos reales, desarrollando tanto conocimiento como habilidades para la gestión como para la implementación de software. 


\begin{tabular}{lll}
\hline Deficiencias & Proceso ISO/IEC 29110 & Beneficios \\
\hline $\begin{array}{l}\text { DC1. Deficiencias en la gestión } \\
\text { de proyectos }\end{array}$ & Proceso de gestión de proyectos & $\begin{array}{l}\text { CDS1-B1, CDS1-B2, CDS1-B3, } \\
\text { CDS1-B4 }\end{array}$ \\
& & $\bullet$ CDS3-B1, CDS3-B2, CDS3-B3 \\
& & CDS4-B2
\end{tabular}

\begin{tabular}{|c|c|c|}
\hline DH1.Toma de decisiones & - Proceso de gestión de proyectos & $\begin{array}{ll}\text { - } & \mathrm{CDS}_{1}-\mathrm{B}_{3} \\
\text { - } & \mathrm{CDS} 2-\mathrm{B}_{4}, \mathrm{CDS} 2-\mathrm{B}_{5} \\
\text { - } & \mathrm{CDS}_{3}-\mathrm{B}_{1}, \mathrm{CDS}_{3}-\mathrm{B}_{2} \\
\text { - } & \mathrm{CDS}_{4}-\mathrm{B}_{3}\end{array}$ \\
\hline
\end{tabular}

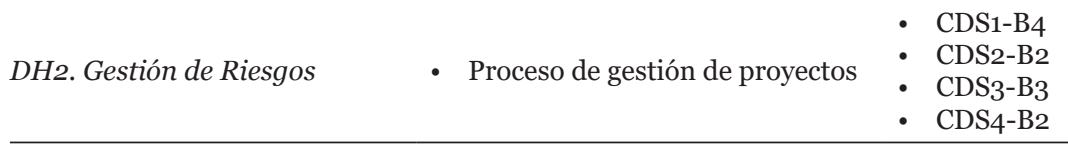

\begin{tabular}{lll}
$\begin{array}{ll}\text { DH3. Capacidad para resolver } \\
\text { problemas }\end{array}$ & $\begin{array}{ll}\text { Proceso de gestión de proyectos } \\
\text { Proceso de implementación del } \\
\text { software }\end{array}$ & $\begin{array}{l}\text { CDS1-B4 } \\
\text { CDS2-B4 }\end{array}$ \\
\hline & & CDS3-B1 \\
\hline
\end{tabular}
DH4. Trabajo en equipo
- Proceso de implementación del software
- $\mathrm{CDS} 1-\mathrm{B}_{3}, \mathrm{CDS} 1-\mathrm{B} 4$
- $\mathrm{CDS}_{2}-\mathrm{B} 5$
- $\mathrm{CDS}_{3}-\mathrm{B}_{4}, \mathrm{CDS}_{3}-\mathrm{B}_{5}$
- $\mathrm{CDS}_{4}-\mathrm{B} 2, \mathrm{CDS}_{4}-\mathrm{B} 3$

Tabla 2 - Mapeo entre deficiencias detectadas en empresas, proceso ISO/IEC 29110 que cubre esa deficiencia y los beneficios identificados en los 4CDS's

Las principales lecciones aprendidas identificadas a partir de la experiencia son:

- Iniciar el proceso identificando y formalizando las buenas prácticas tanto de gestión de proyectos como de implementación de software de los CDS's.

- Proporcionar soporte a los CDS's desde el principio de proceso de adopción del estándar ISO/IEC 29110 y hasta que obtuvieron la certificación.

- Mantener reuniones de seguimiento en las que se brindó retroalimentación a lo largo de todo el proceso. 
- Proporcionar el soporte técnico disponible para los CDS's en cualquier momento que les surgían dudas o preguntas relacionadas con el estándar ISO/IEC 29110 o la implementación de alguna práctica.

Sin embargo, también se han identificado algunas oportunidades de mejora:

- Proporcionar capacitación centrada en la comprensión de la terminología del estándar ISO / IEC 29110.

- Desarrollar herramientas de software que proporcione soporte durante la capacitación estándar ISO / IEC 29110 y su implementación.

\section{Referencias}

ISO. (2012). ISO/IEC TR 29110-5-1-2:2012 Software Engineering-Lifecycle Profiles for Ver Small Entities (VSEs)-Parte 5-1-2: Management and Engineering Guide: Generic Profile Group: Basic Profile. Int'l Org for Standardization.

Laporte, C. Y., Muñoz, M. \& Gerançon, B. (2017). The education of students about ISO/ IEC 29110 software engineering standards and their implementations in very small entities. In: IEEE Canada-International Humanitarian Technology Conference, July 20-21, 2017, Toronto, Ontario, Canada, pp. 94-98.

Laporte, C. Y., Muñoz, M., Mejia, J. \& O’Connor, R.V. (2018). Applying software engineering standards in very small entitites- From Startups to Grownups. IEEE software, 35(1), 99-103.

Laporte, C.Y. \& O’Connor, R.V. (2016). Systems and software engineering standards for very small entities: accomplishments and overview. Computer, IEEE Computer society. 49(8), 84-87

Larrucea, X., O’Connor, R. V., Colomo-Palacios \& R., Laporte, C.Y. (2016). Software Process Improvement in Very Small Organizations. IEEE Software 33(2), 85-89.

Muñoz, M., Peña, A., Mejia, J. \& Lara, G. (2016). Coverage of the university curricula for the Software Engineering industry in Mexico. IEEE Latin America Transactions, 5(14), 2382-2388.

Muñoz, M., Peña A., Mejia, J., \& López, G. L. (2016). Actual State of the Coverage of Mexican Software Industry Requested Knowledge Regarding the Project Management Best Practices. Computer Science and Information Systems, 13(3), 849-873. Retrieved from: https://doi.org/10.2298/CSIS160808040M

Muñoz M., Peña A., Mejia J., \& Lopez G.L. (2018). ISO/IEC 29110 and curricula programs related to Computer Science and Informatics in Mexico: Analysis of practices coverage. In: Mejia J., Muñoz M., Rocha Á., Quiñonez Y., \& Calvo-Manzano J. (eds). Trends and Applications in Software Engineering. CIMPS 2017. Advances in Intelligent Systems and Computing, vol 688. Springer, Cham.

NYCE. (2018). Compañías certificadas en el estándar ISO/IEC 29110-4-1:2011, recuperado de https://www.nyce.org.mx/wp-content/uploads/2018/o2/ PADRON-DE-EMPRESAS-CERTIFICADAS-ISO-IEC-29110-4-1.pdf 
Sánchez-Gordon, M-L., de Amescua, A., O’Connor, R.V. \& Larrueca, X. (2017). A standard-based framework to integrate software work in small settings. Computer Standars \& Interfaces, 54, (Part 3), 162-175.

Software Gurú (2008). Centro de Desarrollo de Software. Un Nuevo paradigma. [En línea]. Disponible en: https://sg.com.mx/content/view/424 